\title{
DISCOVERY OF VERY HIGH ENERGY $\gamma$-RAYS FROM THE BLAZAR S5 0716+714
}

H. Anderhub ${ }^{1}$, L. A. Antonelli ${ }^{2}$, P. Antoranz ${ }^{3}$, M. Backes ${ }^{4}$, C. Baixeras ${ }^{5}$, S. Balestra ${ }^{3}$, J. A. Barrio ${ }^{3}$, D. Bastieri ${ }^{6}$, J. Becerra González ${ }^{7}$, J. K. Becker ${ }^{4}$, W. BednareK ${ }^{8}$, A. Berdyugin ${ }^{9}$, K. Berger $^{8}$, E. Bernardini ${ }^{10}$, A. Biland ${ }^{1}$, R. K. BocK ${ }^{6,11}$, G. BonNoli ${ }^{12}$, P. Bordas ${ }^{13}$, D. Borla Tridon ${ }^{11}$, V. Bosch-RAmon ${ }^{13}$, D. Bose ${ }^{3}$, I. Braun ${ }^{1}$, T. Bretz ${ }^{14}$, D. BritzGer ${ }^{11}$, M. Camara ${ }^{3}$, E. Carmona ${ }^{11}$, A. Carosi ${ }^{2}$, P. Colin ${ }^{11}$, S. Commichau ${ }^{1}$, J. L. Contreras ${ }^{3}$, J. Cortina ${ }^{15}$, M. T. Costado ${ }^{7,16}$, S. Covino ${ }^{2}$, F. Dazzi ${ }^{17,26}$, A. De Angelis ${ }^{17}$, E. de Cea del Pozo ${ }^{18}$, R. De los Reyes ${ }^{3}$, B. De Lotto ${ }^{17}$, M. De Maria ${ }^{17}$, F. De Sabata ${ }^{17}$, C. Delgado Mendez ${ }^{7,27}$, A. Domínguez ${ }^{19}$, D. Dominis Prester ${ }^{20}$, D. Dorner ${ }^{1}$, M. Doro $^{6}$, D. Elsaesser ${ }^{14}$, M. Errando ${ }^{15}$, D. Ferenc ${ }^{21}$, E. FernándeZ ${ }^{15}$, R. FirPo ${ }^{15}$, M. V. FonseCA ${ }^{3}$, L. Font ${ }^{5}$, N. Galante ${ }^{11}$,

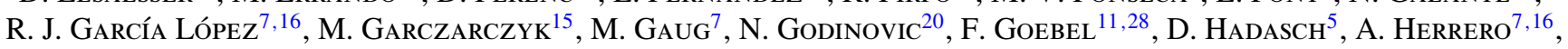

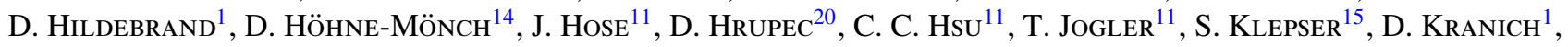

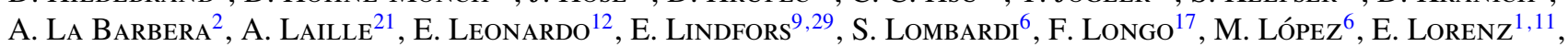

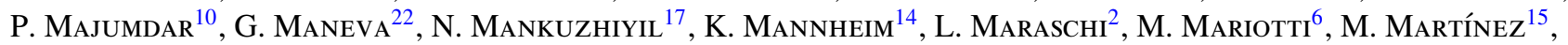
D. Mazin ${ }^{15,29}$, M. Meucci ${ }^{12}$, J. M. Miranda ${ }^{3}$, R. Mirzoyan ${ }^{11}$, H. Miyamoto ${ }^{11}$, J. Moldón ${ }^{13}$, M. Moles ${ }^{19}$, A. Moralejo ${ }^{15}$,

D. Nieto ${ }^{3}$, K. Nilsson ${ }^{9}$, J. Ninkovic ${ }^{11}$, R. Orito ${ }^{11}$, I. Oya ${ }^{3}$, R. Paoletti ${ }^{12}$, J. M. Paredes ${ }^{13}$, M. Pasanen ${ }^{9}$, D. Pascoli ${ }^{6}$, F. Pauss ${ }^{1}$, R. G. Pegna ${ }^{12}$, M. A. Perez-Torres ${ }^{19}$, M. Persic ${ }^{17,23}$, L. Peruzzo ${ }^{6}$, F. Prada ${ }^{19}$, E. Prandini ${ }^{6}$, N. Puchades ${ }^{15}$, I. Puljak ${ }^{20}$, I. Reichardt ${ }^{15}$, W. Rhode ${ }^{4}$, M. Ribó ${ }^{13}$, J. Rico ${ }^{15,24}$, M. Rissi ${ }^{1}$, A. Robert ${ }^{5}$, S. RÜGamer ${ }^{14}$, A. SagGion ${ }^{6}$, J. SAinio ${ }^{9}$,

T. Y. Saito ${ }^{11}$, M. Salvati ${ }^{2}$, M. SÁnchez-Conde ${ }^{19}$, K. Satalecka ${ }^{10}$, V. Scalzotto ${ }^{6}$, V. Scapin ${ }^{17}$, T. Schweizer ${ }^{11}$,

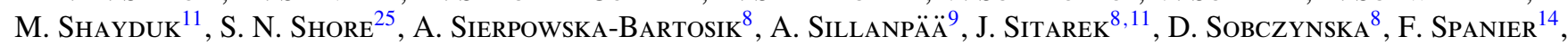
S. Spiro ${ }^{2}$, A. Stamerra ${ }^{12}$, L. S. Stark ${ }^{1}$, T. Suric ${ }^{20}$, L. Takalo ${ }^{9}$, F. Tavecchio ${ }^{2}$, P. Temnikov $^{22}$, D. Tescaro ${ }^{15}$, M. Teshima ${ }^{11}$,

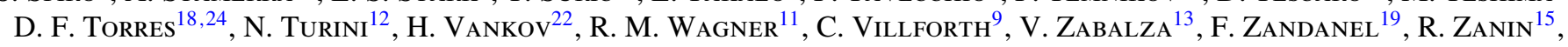
AND J. ZAPATERO 5

${ }^{1}$ ETH Zurich, CH-8093, Switzerland

2 INAF National Institute for Astrophysics, I-00136 Rome, Italy

${ }^{3}$ Universidad Complutense, E-28040 Madrid, Spain

${ }^{4}$ Technische Universität Dortmund, D-44221 Dortmund, Germany

${ }^{5}$ Universitat Autònoma de Barcelona, E-08193 Bellaterra, Spain

${ }^{6}$ Università di Padova and INFN, I-35131 Padova, Italy

${ }^{7}$ Inst. de Astrofísica de Canarias, E-38200 La Laguna, Tenerife, Spain

${ }^{8}$ University of Łódź, PL-90236 Lodz, Poland

${ }^{9}$ Tuorla Observatory, University of Turku, FI-21500 Piikkiö, Finland

${ }^{10}$ Deutsches Elektronen-Synchrotron (DESY), D-15738 Zeuthen, Germany

${ }^{11}$ Max-Planck-Institut für Physik, D-80805 München, Germany

12 Università di Siena, and INFN Pisa, I-53100 Siena, Italy

${ }^{13}$ Universitat de Barcelona (ICC/IEEC), E-08028 Barcelona, Spain

${ }^{14}$ Universität Würzburg, D-97074 Würzburg, Germany

${ }^{15}$ IFAE, Edifici Cn., Campus UAB, E-08193 Bellaterra, Spain

${ }^{16}$ Depto. de Astrofísica, Universidad de La Laguna, E-38206 La Laguna, Tenerife, Spain

${ }^{17}$ Università di Udine, and INFN Trieste, I-33100 Udine, Italy

${ }^{18}$ Institut de Ciències de l'Espai (IEEC-CSIC), E-08193 Bellaterra, Spain

${ }^{19}$ Inst. de Astrofísica de Andalucía (CSIC), E-18080 Granada, Spain

${ }^{20}$ Rudjer Boskovic Institute, Bijenicka 54, HR-10000 Zagreb, Croatia

${ }^{21}$ University of California, Davis, CA 95616-8677, USA

${ }^{22}$ Inst. for Nucl. Research and Nucl. Energy, BG-1784 Sofia, Bulgaria

${ }^{23}$ INAF/Osservatorio Astronomico and INFN, I-34143 Trieste, Italy

${ }^{24}$ ICREA, E-08010 Barcelona, Spain

${ }^{25}$ Università di Pisa, and INFN Pisa, I-56126 Pisa, Italy

Received 2009 July 14; accepted 2009 September 18; published 2009 October 5

\begin{abstract}
The MAGIC Collaboration reports the detection of the blazar S5 $0716+714(z=0.31 \pm 0.08)$ in very high energy gamma rays. The observations were performed in 2007 November and in 2008 April, and were triggered by the Kungliga Vetenskapliga Akademi telescope due to the high optical state of the object. An overall significance of the signal accounts to $S=5.8 \sigma$ for $13.1 \mathrm{hr}$ of data. Most of the signal $(S=6.9 \sigma)$ comes from the 2008 April data sample during a higher optical state of the object suggesting a possible correlation between the Very High Energy $\gamma$-ray and optical emissions. The differential energy spectrum of the 2008 data sample follows a power law with a photon index of $\Gamma=3.45 \pm 0.54_{\text {stat }} \pm 0.2_{\text {syst }}$, and the integral flux above $400 \mathrm{GeV}$ is at the level of $\left(7.5 \pm 2.2_{\text {stat }} \pm 2.3_{\text {syst }}\right) \times 10^{-12} \mathrm{~cm}^{-2} \mathrm{~s}^{-1}$, corresponding to a $9 \%$ Crab Nebula flux. Modeling of the broadband spectral energy distribution indicates that a structured jet model appears to be more promising in describing the available data than a simple one-zone synchrotron self-Compton model.
\end{abstract}

Key words: BL Lacertae objects: individual (S5 0716+714) - gamma rays: observations 


\section{INTRODUCTION}

Blazars, a common term used for flat spectrum radio quasars (FSRQ) and BL Lacertae objects, appear to be the most energetic types of active galactic nuclei (AGNs). In these objects, the dominant radiation component originates in a relativistic jet pointed nearly toward the observer. The double-peaked spectral energy distribution (SED) of blazars is attributed to a population of relativistic electrons spiraling in the magnetic field of the jet. The low-energy peak is due to synchrotron emission and the second, high-energy peak is often attributed to inverse Compton (IC) scattering of low-energy photons in leptonic emission models (Maraschi et al. 1992; Dermer \& Schlickeiser 1993; Bloom \& Marscher 1996). Models based on the acceleration of hadrons can also sufficiently describe the observed SEDs and light curves (Mannheim 1993; Mücke et al. 2003). For most FSRQs and a large fraction of BL Lacertae objects (namely, $\mathrm{LBLs}^{30}$ ), the low-energy peak is located in the energy range between submillimeter and optical. On the other hand, for most of the sources detected to emit VHE $\gamma$-rays $\left(\mathrm{HBLs}^{31}\right)$ the lowenergy peak is located at UV to X-rays energies (Padovani 2007). The high-energy peak is typically at $\mathrm{MeV}-\mathrm{GeV}$ energies. Blazars are highly variable in all wave bands and the relation between variability in different bands is a key element in discriminating between different models.

The MAGIC Collaboration is performing Target of Opportunity observations of sources in a high flux state in the optical and/or X-ray band. Optically triggered observations have resulted in the discovery of VHE $\gamma$-rays from Mrk 180 (Albert et al. 2006) and 1ES 1011+496 (Albert et al. 2007a). In this Letter, we report the results of observations of S5 0716+714 in 2007 November and 2008 April. The observation at the latter date resulted in the discovery of VHE $\gamma$-rays from the source as announced in Teshima et al. (2008).

The BL Lac object S5 0716+714 has been studied intensively at all frequency bands. It is highly variable with rapid variations observed from the radio to X-ray bands (Wagner et al. 1996). It has therefore been target to several multiwavelength campaigns, the most recent one organized by the GLAST-AGILE Support Program in 2007 July-November (Villata et al. 2008; Giommi et al. 2008a). Due to the very bright nucleus, which outshines the host galaxy, the redshift of S5 $0716+714$ is still uncertain. The recent photometric detection of the host galaxy (Nilsson et al. 2008) suggests a redshift of $z=0.31 \pm 0.08$ which is consistent with the redshift $z=0.26$ determined by spectroscopy for three galaxies close to the location of S5 0716+714 (Stickel et al. 1993). In the SED of S5 0716+714, the synchrotron peak is located in the optical band and is, therefore, classified either as LBL (Nieppola et al. 2006) or as IBL ${ }^{32}$ (Padovani \& Giommi 1995).

S5 $0716+714$ was detected in the $\mathrm{MeV}$ energy range several times at different flux levels by the EGRET detector on board the Compton Gamma-ray Observatory (Hartman et al. 1999). In 2008, AGILE reported the detection of a variable $\gamma$-ray flux with a peak flux density above the maximum reported

\footnotetext{
${ }^{26}$ Supported by INFN Padova.

${ }^{27}$ Now at Centro de Investigaciones Energéticas, Medioambientales y Tecnológicas.

${ }^{28}$ Deceased.

${ }^{29}$ Send reprint requests to Elina Lindfors elilin@utu.fi and Daniel Mazin mazin@ifae.es

${ }^{30}$ LBL: low frequency peaking BL Lacertae.

31 HBL: high frequency peaking BL Lac.

32 IBL: intermediate frequency peaking BL Lacertae.
}
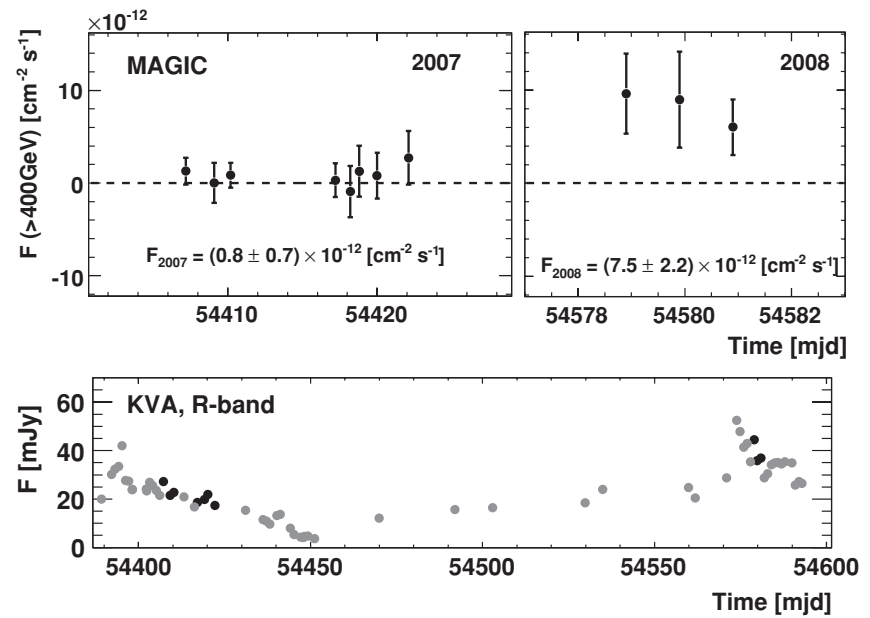

Figure 1. Light curve of S5 $0716+714$ as measured from 2007 November until 2008 April. The day-by-day $\gamma$-ray light curve from MAGIC is shown in the upper panel for the 2007 data (left panel) and 2008 data (right panel), whereas the optical KVA data are shown in the lower panel. The simultaneous optical with the MAGIC data are marked black. The error bars $(1 \sigma)$ of the optical fluxes are smaller than the points and thus not visible.

from EGRET (Chen et al. 2008). S5 0716+714 is also on the Fermi-LAT bright source list (Abdo et al. 2009). Observations at VHE $\gamma$-ray energies by HEGRA resulted in an upper limit of $F(>1.6 \mathrm{TeV})=3.13 \times 10^{-12}$ photons $\mathrm{cm}^{-2} \mathrm{~s}^{-1}$ (Aharonian et al. 2004). In this Letter, we present the first detection of VHE $\gamma$-rays from S5 $0716+714$. It is the third optically triggered discovery of a VHE $\gamma$-ray emitting blazar by MAGIC.

\section{OBSERVATIONS}

The MAGIC telescope is a standalone imaging atmospheric Cherenkov telescope located on the Canary Island of La Palma. MAGIC has a standard trigger threshold of $60 \mathrm{GeV}$ for observations at low zenith angles, an angular resolution of $\sim 0.1$ on the event by event basis and an energy resolution above $150 \mathrm{GeV}$ of $\sim 25 \%$ (see Albert et al. 2008a, for details).

The Tuorla blazar monitoring program ${ }^{33}$ (Takalo et al. 2007) monitors S5 $0716+714$ on a nightly basis using the KVA $35 \mathrm{~cm}$ telescope at La Palma ${ }^{34}$ and the Tuorla $1 \mathrm{~m}$ telescope. At the end of 2007 October (22, MJD = 54395), the optical flux had more than doubled (from $19 \mathrm{mJy}$ to $42 \mathrm{mJy}$ ) in less than a month and, according to a predefined criteria MAGIC was alerted. Due to moon and weather constraints, the MAGIC observations started 11 days later, when the optical flux had already decreased significantly (see Figure 1). The observations were performed in the wobble mode (Daum et al. 1997) pointing 0.4 offset from the source to enable simultaneous estimation of the background from the same field of view. MAGIC observed the source during 14 nights for a total of $16.8 \mathrm{hr}$. During some nights, the observing conditions were rather poor and the affected data were rejected from the analysis. The exposure time for good quality data amounts to $10.3 \mathrm{hr}$. The zenith angle range of these observations was from $42^{\circ}$ to $46^{\circ}$.

In 2008 April, a new bright and fast optical flare occurred. The optical flux almost doubled within three nights (April 14, MJD = 54570: 29 mJy, April 17: $52 \mathrm{mJy}$ ), and at April 17 reached its historical maximum value. MAGIC started the observations five nights later, when the moon conditions allowed. The source was

\footnotetext{
33 http://users.utu.fi/kani/1m

34 http://tur3.tur.iac.es/
} 


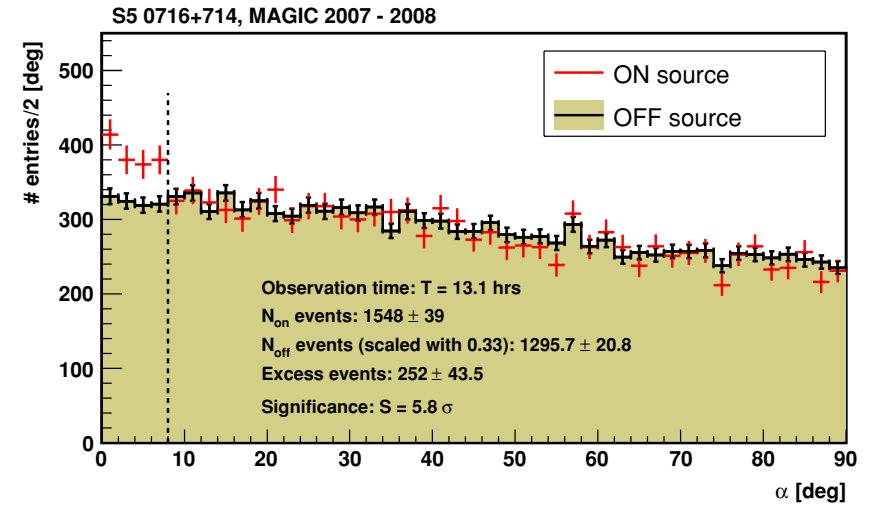

Figure 2. $\mid$ ALPHA $\mid$ distribution after all cuts for the total MAGIC data sample in 2007-2008. A $\gamma$-ray excess with a significance of $5.8 \sigma$ is found.

observed during nine nights with zenith angles from $47^{\circ}$ to $55^{\circ}$ for a total of $7.1 \mathrm{hr}$. Unfortunately, during the last six nights of the observations, there was strong calima wind carrying fine sand from Sahara desert and these data were, therefore, of bad quality and rejected from the analysis. The total exposure time of good quality data for this observation period amounts to only $2.8 \mathrm{hr}$. The total live time of S5 0716+714 MAGIC observations in 2007 and 2008 after data quality cuts was $13.1 \mathrm{hr}$.

\section{DATA ANALYSIS AND RESULTS}

The MAGIC data were analyzed using the standard analysis chain as described in Albert et al. (2008a, 2008b) and Aliu et al. (2009). In order to suppress the background showers produced by charged cosmic rays, a multivariate classification method known as Random Forest is used (Albert et al. 2008c). For every event, the algorithm takes as input a set of image parameters, and produces one single parameter as output, called HADRONNESS. The background rejection is then achieved by a cut in HADRONNESS, which was optimized using Crab Nebula data taken under comparable conditions.

The cut in ALPHA $\mid$ that defines the signal region was also optimized in the same way. An additional cut removed the events with a total charge of less than 200 photoelectrons (phe) in order to assure a better background rejection. We used standard cuts in HADRONNESS and |ALPHA|, which are determined to give the best significance for a point-like source with a flux on the $10 \%$ level of the Crab Nebula flux. For the given cuts and the relatively large zenith angles of the observations, the analysis threshold corresponds to $400 \mathrm{GeV}$. The resulting |ALPHA| distribution after all cuts for the overall S5 0716+714 data sample in 20072008 is shown in Figure 2. An overall excess of $252 \gamma$-like events corresponding to a significance of $S=5.8 \sigma$ was found (following Equation (17) in Li \& Ma (1983); $N_{\text {on }}=1548, N_{\text {off }}$ $=3887$, alpha $=0.33)$. Most of the signal comes from the 2008 data sample: the analysis of the 2008 data alone results in 176 excess events over 422 background events corresponding to $S=6.9 \sigma$. From the 2007 data alone, an excess corresponding to $S=2.2 \sigma$ was found.

The day-by-day light curve as measured by MAGIC data is shown in Figure 1 (upper panel) together with the optical KVA light curve (lower panel). In 2007 November, the MAGIC flux above $400 \mathrm{GeV}$ is at $F_{2007}(>0.4 \mathrm{TeV})=\left(0.8 \pm 0.7_{\text {stat }} \pm\right.$ $\left.0.2_{\text {syst }}\right) \times 10^{-11}\left(\mathrm{~cm}^{-2} \mathrm{~s}^{-1}\right)$, whereas the flux is about nine times higher in 2008: $F_{2008}(>0.4 \mathrm{TeV})=\left(7.5 \pm 2.2_{\text {stat }} \pm\right.$ $\left.2.3_{\text {syst }}\right) \times 10^{-11}\left(\mathrm{~cm}^{-2} \mathrm{~s}^{-1}\right)$. No significant variability is seen on timescales shorter than six months. Given the limited effective

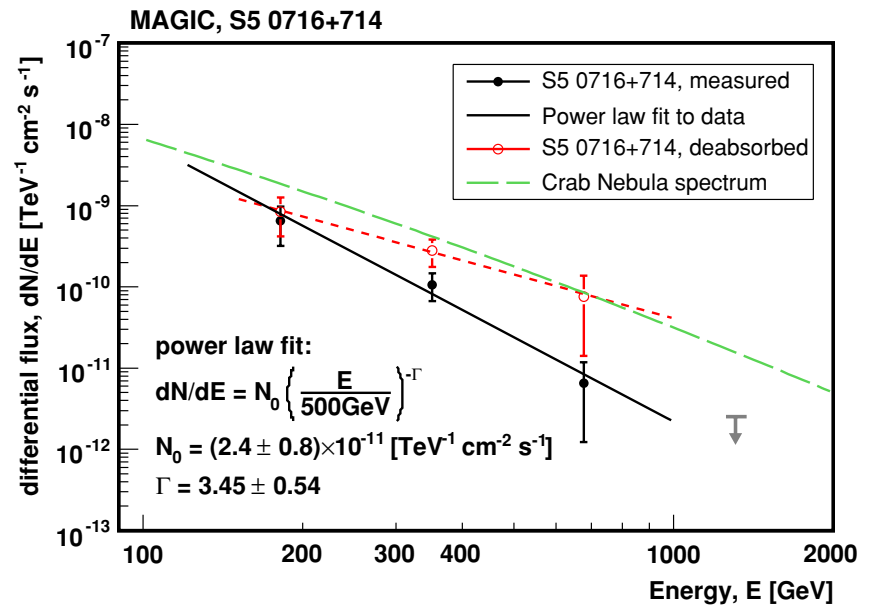

Figure 3. Differential energy spectrum of S5 0716+714. Only data from 2008 April are used. The measured (black points) as well as deabsorbed (using the EBL model of Franceschini et al. (2008) and assuming $z=0.26$, red points) spectra are shown. The results from a power-law fit to the measured spectrum are shown in the plot. The Crab Nebula spectrum is shown for comparison (green dashed line).

exposure times, these observations are not sensitive to variability on shorter timescales. The individual MAGIC points would have low significance, and an intra-night variability by a factor of at least 10 would have been required to detect it. In the optical band, instead, a clear variability on timescales from days to months is visible with two distinct flares: the first in 2007 October and the second in 2008 April (Figure 1, lower panel).

The differential energy spectrum is calculated only for the 2008 April data set. The measured and unfolded for detector effects (Albert et al. 2007c) spectrum is shown in Figure 3. The data point at $E=1.3 \mathrm{TeV}$ has a significance below $1 \sigma$ and was, therefore, converted into an upper limit corresponding to a $95 \%$ confidence level. The measured spectrum can be well fitted by a simple power law (with the differential flux given in units of $\mathrm{TeV}^{-1} \mathrm{~cm}^{-2} \mathrm{~s}^{-1}$ )

$$
\frac{d N}{d E d A d t}=(2.4 \pm 0.8) \times 10^{-11}(E / 500 \mathrm{GeV})^{-3.5 \pm 0.5}
$$

The errors are statistical only. The systematic uncertainties are estimated to be 0.2 on the photon index and $30 \%$ on the absolute flux level. Due to the energy-dependent attenuation of VHE $\gamma$ rays with low-energy photons of the extragalactic background light (EBL; Gould \& Schréder 1967), the VHE $\gamma$-ray flux of distant sources is significantly suppressed. We calculated the deabsorbed, i.e., intrinsic, spectrum of S5 0716+714 using an EBL model of Franceschini et al. (2008) and assuming a redshift of $z=0.26$. The resulting intrinsic spectrum (shown in Figure 3, red points) has a fitted photon index of $\Gamma=1.8 \pm 0.6$, which is well within the range of other extragalactic sources measured so far.

As the source redshift is still uncertain, we used the MAGIC spectra to calculate upper limits to the redshift. We assumed two different maximum values for a possible hardness of the intrinsic spectrum: 1.5 , being a canonical value for a $\gamma$-ray spectrum emitted by electrons with a spectral index of 2.0 and 0.666 , being the limiting case for a $\gamma$-ray spectrum emitted by a monoenergetic electron distribution. Using the method described in Mazin \& Goebel (2007), we obtain the following upper limits for the redshift: $z<0.46$ (for an assumed intrinsic 


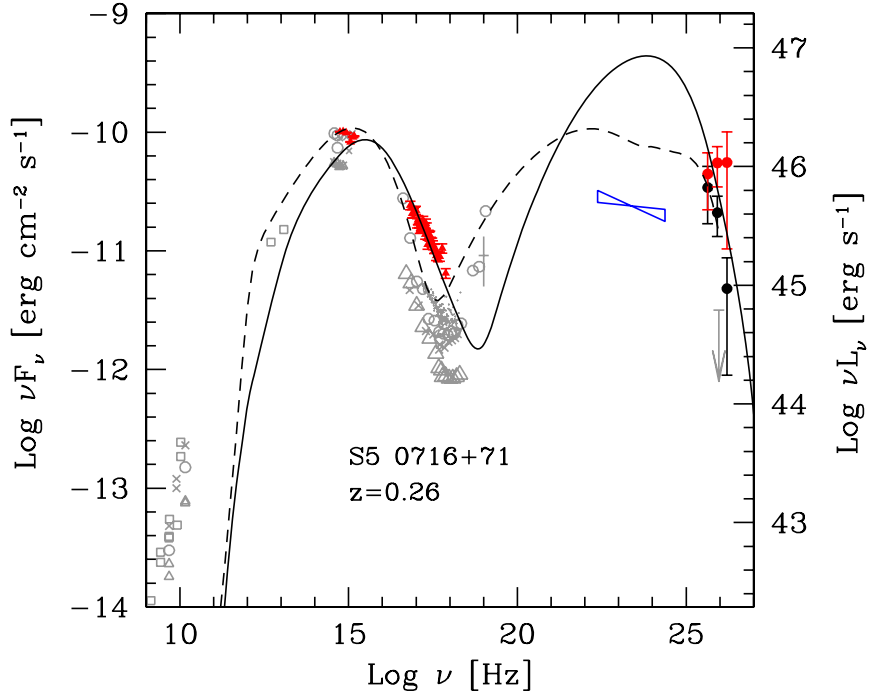

Figure 4. Broadband SED of S5 0716+714. In red is the KVA (from 2008 April 23), Swift/UVOT and Swift/XRT (from 2008 April 29), and the deabsorped MAGIC (from 2008 April 22 to 2008 April 24) data. The MAGIC measured flux for 2008 April is shown in black and the MAGIC upper limit for 2007 November is shown with gray arrow. The gray symbols show historical data (see Tavecchio \& Ghisellini 2009). The bow tie shows the flux from the Fermi bright list. The solid line shows the overall emission calculated with a one-zone SSC model using the 2008 April data (red), see the text for model parameters. The dashed line shows the emission calculated with the spine-layer model described in Tavecchio \& Ghisellini (2009). We assume the same parameters reported there, but a volume three times larger.

spectrum with a power-law photon index of 1.5) and $z<0.59$ (for an assumed intrinsic spectrum with a power-law photon index of 2/3). Both limits agree with the redshift determined from the host galaxy detection $(z=0.31 \pm 0.08)$ and from the spectroscopy of three nearby galaxies $(z=0.26)$.

\section{DISCUSSION}

MAGIC observed the blazar S5 0716+714 in 2007 November and 2008 April, the observations resulting in the discovery of a very high energy $\gamma$-ray excess with a significance of $5.8 \sigma$. During the 2007 November MAGIC observations, the average optical flux was $\sim 20 \mathrm{mJy}$, while in 2008 April the optical flux was $\sim 45 \mathrm{mJy}$. The same trend is also visible in the MAGIC data: the flux in 2008 April is significantly higher than in 2007 November. This seems to support the indication seen in previous MAGIC observations for other BL Lac objects Albert et al. (2006, 2007a, 2007b), that there is a connection between optical high states and VHE $\gamma$-ray high states.

In 2008 April, S5 0716+714 was also in a historical high state in X-rays (Giommi et al. 2008b) and the optical polarization angle started to rotate immediately after the optical maximum had been reached (Larionov et al. 2008). However, the radio flux at $37 \mathrm{GHz}$ did remain at a quiescent level (A. Lähteenmäki 2008, private communication). This energy-dependent behavior is very similar to the one seen in BL Lac in 2005, which was attributed to an emission feature moving in a helical path upstream of the Very Long Baseline Army (VLBA) core (Marscher et al. 2008). The high VHE $\gamma$-ray flux of S5 0716+714 observed by MAGIC might originate from such a moving emission feature, but a more detailed study of the multiwavelength light curves from Spring 2008 are needed to confirm this.

We tried modeling the SED with a one-zone synchrotron selfCompton (SSC) model with relativistic electrons following a smoothed broken power-law energy distribution (see Tavecchio et al. 2001 for a full description). The fit parameters are the minimum electron energy $\gamma_{\min }=10^{4}$, the break energy in the electron spectrum $\gamma_{b}=1.9 \times 10^{4}$, the maximum electron energy $\gamma_{\max }=7 \times 10^{5}$, the electron spectral index below and above the break energy $n_{1}=2, n_{2}=4.5$, respectively, the magnetic field $B=0.13 \mathrm{G}$, the normalization factor $K=1.4 \times 10^{5}$ particles $\mathrm{cm}^{-3}$, the radius of the emitting region $R=7 \times 10^{15} \mathrm{~cm}$, and the Doppler factor $\delta=30$. To account for the observed $\mathrm{TeV}$ flux corrected for intergalactic absorption, the model (continuous line in Figure 4) predicts a high intensity peak in the $10 \mathrm{GeV}$ range. Although the source was flaring in optical at the time of the MAGIC detection, such high $\gamma$ ray flux (more than 10 times larger than observed by EGRET and Fermi, open blue bow tie in Figure 4) appears somewhat implausible. Therefore, we also considered the emission from a structured jet, modeled as a fast "spine" surrounded by a slower moving "layer" (Ghisellini et al. 2005). Photons emitted by the layer are subject to IC scattering by the relativistic electrons of the spine thus introducing an additional contribution to the IC spectrum at energies higher than SSC can reach. The computed model is shown as a dashed line in Figure 4. The GeV excess is substantially reduced and the spectral shape in the $\gamma$-ray domain is consistent with the spectral indices measured at both $\mathrm{GeV}$ and $\mathrm{TeV}$ energies, though with presumably different intensity states.

The discovery of high flux of VHE $\gamma$-rays by MAGIC from the blazar S5 0716+714 in 2008 April should be compared to detailed light curves from other wavelengths (radio, optical, and $\mathrm{X}$-rays) as well as VLBA maps and optical polarization light curve in order to further investigate the origin of this interesting event.

We thank the Instituto de Astrofisica de Canarias for the excellent working conditions at the Observatorio del Roque de los Muchachos in La Palma. The support of the German BMBF and MPG, the Italian INFN and Spanish MICINN is gratefully acknowledged. This work was also supported by ETH Research Grant TH 34/043, by the Polish MNiSzW Grant N N203 390834, and by the YIP of the Helmholtz Gemeinschaft. The authors also thank the anonymous referee for the valuable comments and suggestions, which helped to improve the Letter.

\section{REFERENCES}

Abdo, A., et al. (The Fermi LAT Collaboration) 2009, ApJ, 700, 597 Aharonian, F., et al. (The HEGRA Collaboration) 2004, A\&A, 421, 529 Albert, J., et al. (The MAGIC Collaboration) 2006, ApJ, 648, L105 Albert, J., et al. (The MAGIC Collaboration) 2007a, ApJ, 667, L21 Albert, J., et al. (The MAGIC Collaboration) 2007b, ApJ, 666, L17 Albert, J., et al. (The MAGIC Collaboration) 2007c, Nucl. Instrum. Methods A, 583,494

Albert, J., et al. (The MAGIC Collaboration) 2008a, ApJ, 674, 1037

Albert, J., et al. (The MAGIC Collaboration) 2008b, Nucl. Instrum. Methods A, 594,407

Albert, J., et al. (The MAGIC Collaboration) 2008c, Nucl. Instrum. Methods A, 588,424

Aliu, E., et al. (The MAGIC Collaboration) 2009, Astropart. Phys., 30, 293 Bloom, S. D., \& Marscher, A. P. 1996, ApJ, 461, 657

Chen, A. W., et al. 2008, A\&A, 489, 37

Daum, A., et al. (The HEGRA Collaboration) 1997, Astropart. Phys., 8, 1

Dermer, C. D., \& Schlickeiser, R. 1993, ApJ, 416, 458

Franceschini, A., Rodighiero, G., \& Vaccari, M. 2008, A\&A, 487, 837

Giommi, P., et al. 2008a, A\&A, 487, 49

Giommi, P., et al. 2008b, ATel 1495

Ghisellini, G., Tavecchio, F., \& Chiaberge, M. 2005, A\&A, 432, 401

Gould, R. J., \& Schréder, G. P. 1967, Phys. Rev., 155, 1408

Hartman, R. C., et al. 1999, ApJS, 123, 79 
Larionov, V., et al. 2008, ATel, 1502

Li, T.-P., \& Ma, Y.-Q. 1983, ApJ, 272, 317

Mannheim, K. 1993, A\&A, 269, 67

Maraschi, L., Ghisellini, G., \& Celotti, A. 1992, ApJ, 397, L5

Marscher, A. P., et al. 2008, Nature, 452, 966

Mazin, D., \& Goebel, F. 2007, ApJ, 655, L13

Mücke, A., et al. 2003, Astropart. Phys., 18, 593

Nieppola, E., Tornikoski, M., \& Valtaoja, E. 2006, A\&A, 445, 441

Nilsson, K., et al. 2008, A\&A, 487, L29

Padovani, P. 2007, Ap\&SS, 309, 63
Padovani, P., \& Giommi, P. 1995, MNRAS, 277, 1477

Stickel, M., Fried, J. W., \& Kuehr, H. 1993, A\&AS, 98, 393

Takalo, L. O., et al. 2007, in ASP Conf. Ser. 373, The Central Engine of Active Galactic Nuclei, ed. Luis C. Ho \& Jian-Min Wang (San Francisco, CA: ASP), 249

Tavecchio, F., \& Ghisellini, G. 2009, MNRAS, 394, 131

Tavecchio, F., et al. 2001, ApJ, 554, 725

Teshima, M. (for the MAGIC Collaboration) 2008, ATel, 1500

Villata, M., et al. 2008, A\&A, 480, 339

Wagner, S. J., et al. 1996, AJ, 111, 2187 